\title{
The Relative Importance of Niche Process and Neutral Process in the Community Assembly of Subtropical Karst Forest: A Perspective on the Changes of Species Distribution Patterns at Different Scales
}

\author{
Yan He \\ Guangxi Normal University https://orcid.org/0000-0003-1844-0808 \\ Yong Jiang ( $\sim$ Yongjiang226@126.com ) \\ Guangxi Normal University \\ Hongling Lin \\ Guangxi Normal University \\ Yuanfang Pan \\ Guangxi Normal University \\ Shichu Liang \\ Guangxi Normal University \\ Yaocheng Fang \\ Guangxi Normal University \\ Wenhua Zhuo \\ Guangxi Normal University \\ Yanmei Xiao \\ Guangxi Normal University
}

\section{Research Article}

Keywords: Karst, Community assembly, Niche process, Neutral process, Species abundance distribution pattern

Posted Date: November 8th, 2021

DOI: https://doi.org/10.21203/rs.3.rs-1035471/v1

License: (c) (i) This work is licensed under a Creative Commons Attribution 4.0 International License.

Read Full License 
1 The relative importance of niche process and neutral process in the community

2 assembly of subtropical karst forest: a perspective on the changes of species

3 distribution patterns at different scales

4 Yan He $\cdot$ Yong Jiang $(\bowtie) \cdot$ Hongling Lin $\cdot$ Yuanfang Pan $\cdot$ Shichu Liang $\cdot$ Yaocheng Fang $\cdot$ Wenhua

5 Zhuo $\cdot$ Yanmei Xiao

6 College of Life Science, Guangxi Normal University, Guilin 541006, China

7 Key Laboratory of Wild Animal and Plant Ecology Guangxi Colleges and Universities, Guangxi

$8 \quad$ Normal University, Guilin 541006, China

9 E-mail: yongjiang226@126.com

\section{Abstract}

Background and aims

13 The importance of niche processes and neutral processes to community assembly has been affirmed by most studies, although their relative importance needs to be determined in many systems. Moreover, as the spatial scale changes, the ecological processes that determine the community pattern may differ. We tested these ideas in subtropical karst forest in southwestern China in order to aid efforts of community reconstruction.

Methods

To test the importance of niche-based and neutral mechanisms we compared the fit six models to the observed SAD of the plot at three different sampling scales $(10 \mathrm{~m} \times 10 \mathrm{~m}, 20 \mathrm{~m} \times 20 \mathrm{~m}, 50 \mathrm{~m} \times 50 \mathrm{~m})$. We also used spatial autocorrelation and distance-based Moran's eigenvector maps (dbMEM) combined with variation partitioning to further determine the relative contribution of the niche process and the neutral process under different sampling scales.

24 Results

The neutral theoretical and statistical models fit the observed species abundance distribution curve best at each sampling scale. And variation partitioning showed that although the contribution of spatial structure was lower at larger sampling scales, it was still important, suggesting that neutral processes drive community structure at all sampling scales. In contrast, habitat filtering and interspecies competition may lead to a net weakening of the contribution of the niche process to the species abundance pattern of the community because they act in opposite directions.

31 Conclusions

32 In the restoration and reconstruction of local karst forest communities, environmental heterogeneity, inter-species relationships, and geographic spatial differences should all be considered. 
Keywords Karst; Community assembly; Niche process; Neutral process; Species abundance distribution pattern

\section{Introduction}

Understanding the mechanisms that drive the variation and maintenance of species diversity in ecological communities remains a fundamental question in biology. However, the effect of ecological processes on the community is almost impossible to directly observe, so it is an effective way to infer potential ecological processes from the observed patterns. The species abundance distribution (SAD) describes the ranked abundance of species within a community, and is commonly used to describe community structure and species diversity in ecological research (Preston, 1948; Magurran, 2004; Li et al., 2015). Examining SADs has become a powerful tool for describing community structure and pattern (McGill et al., 2007) because species abundance distribution curves can intuitively express characteristics of community structure, such as the relative proportion of rare and common species at any one site, which is convenient for comparing ecological communities with different properties. It is also an important means to reveal community structure and the regional distribution of species (Ma, 2003). Studying patterns of species abundance distribution is also of great significance in the protection and management of biodiversity. For example, the rarity of species is an important reference for determining the objectives of biodiversity protection, and the characteristics of the SAD are sometimes regarded as an indicator of the "healthiness" of a community (Izsák \& Pavoine, 2012; Matthews \& Whittaker, 2015).

The structure of ecological communities is the result of the combined effects of various ecological processes that are assumed to leave signals on patterns of species distribution (Hubbell, 2001). Therefore, the SAD curve of a community has been widely used in attempts to detect the effects of niche differentiation, seed dispersal limitation, species differentiation, extinction mechanisms, and processes driving community structure and dynamics (Green, 2007). Dozens of models, based on different theories, have been used to fit and interpret SAD patterns. In general, these models can be divided into statistical models and mechanistic models. Pure statistical models provide an empirical fitting of the species abundance distribution, but their biological and ecological significance is not clear. For example the commonly used log-series (Fisher et al., 1943) and log-normal models (Preston, 1948) reflect more mathematical distribution laws than ecological significance.

In contrast, mechanistic models attach importance to the biological significance of the model, and different models reflect different ecological processes. Mechanistic models can be further divided into neutral theory models and niche models. According to the assumption of community saturation, the neutral theory hypothesizes that the distribution pattern of species abundance in local communities obeys a zero-sum multinomial distribution, for example the model of Hubbell (2001). This model assumes that the local community has many rare species, but the specific number is affected by the community size and dispersal limitation. In contrast, models based on niche theory have also been developed to fit the SAD, including the broken stick, niche preemption, overlapping niche, dominance decay, and power fraction models (MacArthur, 1957; Tokeshi, 1993; Tokeshi, 1996). These models differ in the niche segmentation mode of species. Therefore, comparing the fit of a suite of alternative 
models to actual observed species abundance distribution patterns can help infer the relative importance of the underlying mechanism/s (Zhang et al., 2016; Furniss et al., 2017; Wu et al., 2019).

It is well known that niche theory and neutral theory differ in their assumptions concerning the factors that influence the species composition of a community. The former assumes that species composition is affected by inter-species competition and environmental variation, while the latter suggests that the distribution pattern of species is affected by dispersal limitation and that therefore species composition changes with space or distance (Niu et al., 2009; Chai \& Yue, 2016). The relative importance of environmental variation versus spatial effects is increasingly examined using redundancy analysis to perform variation partitioning on patterns of species composition. By comparing the relative explanatory power of environmental and spatial factors to species composition, we can infer the relative importance of habitat filtering (based on niche theory) and dispersal limitation (based on neutral theory) (Legendre et al., 2009; Vellend et al., 2014). Similarly, spatial autocorrelation analysis can be used to test whether the specific SAD of a community is significantly related to the SAD of neighboring communities (Kong et al., 2011; Wang et al., 2014). While previous work focused on the primacy of niche theory versus neutral theory, a more productive approach is to examine their relative contrbutions to mechanisms of the maintenance of species diversity (Gravel et al., 2006; Zillio and Condit, 2007; Zhang et al., 2016).

In addition, the relative importance of these ecological processes that determine community structure may vary at different spatial scales, leading to variation in the SAD across spatial scales. Community habitat heterogeneity, $\beta$ diversity, degree of individual aggregation, and spatial autocorrelation will increase correspondingly with the increase in sampling size (McGill et al., 2007), and these factors may cause different distribution patterns. For example, the importance of habitat filtering is more pronounced at large sampling scales, while the effect of interspecies competition is more pronounced on small sampling scales (Shipley et al., 2012; Wiegand et al., 2017). Further, the species abundance distribution gradually changes to the log left skew as spatial scale increases, likely caused by spatial autocorrelation (McGill 2003). Therefore, the pattern and process of community biodiversity and the mechanism of biodiversity maintenance are closely related to the sampling scale of the studied community (Hubbell \& Borda-De-Água, 2004).

We addressed these ideas in the typical karst forest community of evergreen and deciduous broadleaved mixed forest in Guilin, Guangxi, southwestern China with the aim to better understand mechanisms of community structure and provide a theoretical basis for the restoration and reconstruction of vegetation in the area. We established a medium-sized forest plot, and compared the fit of a suite of statistical, niche, and neutral models to the observed SAD. Then, we used variance partitioning to assess the relative importance of environmental variation and space to community structure and SAD. If niche processes primarily drive the SAD here, we should see that the pure environmental components or the spatial structure of the environment occupy a larger explanatory ratio. In contrast, if neutral processes are more important, we should see that simple spatial components occupy a larger explanatory ratio.

\section{Material and methods}

Study system 
Evergreen and deciduous broad-leaved mixed forest is typical vegetation of the subtropical karst region of China, in a transition zone from deciduous broad-leaved forest to evergreen deciduous broad-leaved forest. It has the characteristics of rich biodiversity, complex community structure, and significant ecological benefits (Wu et al., 2019). The global area of karst is nearly 22 million $\mathrm{km}^{2}$, mainly in lowlatitude areas. The concentrated and contiguous areas mainly include three large areas in central and southern Europe, eastern North America, and southwest China. In Europe and North America, the small vulnerability of the geological environment and relatively small population and economic power mean that the ecological and geological environmental problems in karst forest are not very serious. However, the karst in southwestern China is known for its large continuous distribution and extensive human development, as well as a fragile ecological environment ( $\mathrm{Li}$ et al., 2004). As such, rocky desertification has become the most serious ecological geo-environmental problem that restricts the sustainable development in karst of southwest China (Lei et al., 2014). Research on these issues is urgently needed.

Due to the harsh abiotic environment of subtropical karst, niche differentiation is often regarded as the most important process of community construction in these forests (Yao et al., 2020). However, dispersal is the first step in community construction, and the composition of the initial species in the community strongly affects the entry and settlement of subsequent species (Wang et al., 2013). With the succession of the community, when the karst forest succeeds to the relatively stable stage of the evergreen and deciduous broad-leaved mixed forest, the relative importance of niche processes and neutral processes to its community construction is still unknown.

Study area

The study area is located in the karst area of Guilin, Guangxi Zhuang Autonomous Region, China. This area belongs to the mid-subtropical humid monsoon climate, with abundant rainfall and mild climate. It has obvious characteristics of karst areas. The annual average temperature is $18-19^{\circ} \mathrm{C}$, the average temperature in the coldest January is $8^{\circ} \mathrm{C}$, and the average temperature in the hottest August is $28^{\circ} \mathrm{C}$. The annual frost-free period is 309 days, and the annual rainfall is $1,856 \mathrm{~mm}$. The rainfall distribution throughout the year is uneven: Spring and summer are humid and rainy. The annual average evaporation is 1,458 mm (Liu et al., 2020; Pan et al., 2021). The main vegetation type is subtropical evergreen and deciduous broad-leaved mixed forest.

Community survey and environmental factor measurement

In this study, a typical karst evergreen and deciduous broad-leaved mixed forest community $\left(24^{\circ} 59^{\prime} 57.23^{\prime \prime}-24^{\circ} 59^{\prime} 55.72^{\prime \prime} \mathrm{N}, 110^{\circ} 07^{\prime} 25.87^{\prime \prime}-110^{\circ} 07^{\prime} 18.49^{\prime \prime} \mathrm{E}\right)$, was selected as the research object to establish and investigate the plot. It is located in Guilin, Guangxi Zhuang Autonomous Region, China. Following standard ForestGeo protocols (https://forestgeo.si.edu/), we established a fixed monitoring plot of $200 \mathrm{~m} \times 100 \mathrm{~m}$. All woody plants with a diameter at breast height (DBH, $1.3 \mathrm{~m}) \geq 1 \mathrm{~cm}$ in the plot were enumerated, mapped, marked, and identified. We recorded their species scientific name, position, DBH, and height. For subsequent analyses, we used three sizes of subplots: $10 \times 10 \mathrm{~m}(\mathrm{n}=$ $200), 20 \times 20 \mathrm{~m}(\mathrm{n}=50)$ and $50 \times 50 \mathrm{~m}(\mathrm{n}=8)$.

We obtained interpolated data on several environmental factors within the study plot, including 
Chinese Soil Society, 1983). Fresh soil samples were collected and placed into Ziplock bags for transport to the laboratory. A total of seven physical and chemical properties of the soils samples were measured: soil $\mathrm{pH}(\mathrm{pH})$, organic matter (SOM), total nitrogen $(\mathrm{TN})$, available nitrogen (AN), total phosphorus (TP), available phosphorus (AP), and available potassium (AK).

Topographical factors included elevation, slope, convexity, and aspect. For each sample square, elevation was calculated as the average of the elevation at the four vertices of the sample square. Slope was calculated as the average of the angles between the plane and the horizontal plane formed by any three angles of the target sample square. The elevation of the target square minus the average elevation of the surrounding eight squares was used as the concavity and convexity of each sample square. For a sample square located at the edge or four corners of the sample plot, its convexity value is obtained by subtracting the average elevation of several adjacent sample squares from the elevation of the sample square (Harms et al, 2001; Valencia et al, 2004). The aspect of each sample square was calculated using the following formula:

$$
\text { Aspect }=180-\arctan \left(\frac{f y}{f x}\right) \times\left(\frac{180}{3.14}\right)+90 \times(f x /|f x|) .
$$

where $f x$ and $f y$ are the difference in elevation of the sample square from east to west and north to south, respectively. The aspect is a circular variable with a range of $[0,2 \pi]$, so it needs to be converted into a sine and cosine to be a linear variable and included in the subsequent analysis.

\section{Statistical analyses}

To generate the species abundance distributions, we counted the abundance of each trees $>1 \mathrm{~cm}$ DBH for each species within each sample square and then sorted them according to their relative abundance from high to low. The species with the highest abundance had a level of 1, the species with the secondhighest abundance had a level of 2, and so on. The average value of species abundance at each level was used as the final species abundance value under the sampling scale. To obtain the species abundance curve for each sampling scale, we plotted the $\log 10$ abundance as a function of their abundance index.

In order to assess the relative importance of niche processes and neutral processes to community assembly, we used three types of model to explain and quantify the patterns and processes of the species abundance distribution: two niche models (broken stick model and niche preemption model), two statistical models (log-normal model and log-series model), and two neutral theory models (metacommunity zero-sum multinomial distribution model and Volkov model). A brief introduction to the fitted models used follows below.

Niche models

The broken stick model, proposed by MacArthur (1957), is a resource allocation model. The model assumes that the total niche (total resources) in a community is equivalent to a stick equal to 1 . Resources are allocated by randomly setting $\mathrm{S}-1$ points on the stick, and dividing the stick into $\mathrm{S}$ segments, representing the niches occupied by $\mathrm{S}$ species. The model assumes that $\mathrm{S}$ species have similar taxonomic status, similar competitive abilities, and appear in the community at the same time. The total number of individuals in the community is $\mathrm{J}$. Then the abundance of the $i$-th species in the model is: 


$$
A_{i}=\frac{J}{S} \sum_{x=i}^{S} \frac{1}{x}(i=1,2,3, \ldots, S) .
$$

The niche preemption model assumes that the most dominant species in the community first occupies $\mathrm{k}$ shares of the total niche, and the subdominant species occupies the remaining $\mathrm{k}$ shares, namely $\mathrm{k}(1-\mathrm{k})$, and so on, until the remaining resources can no longer sustain a species (Motonura, 1932; Cheng et al., 2011; Zhang et al., 2015). This model guarantees that dominant species have priority in the use of resources, and all species clearly form a hierarchy in niche occupation. Here, let $\mathrm{A}_{1}$ represent the abundance of the most dominant species in the model, then the abundance of the $i$-th species in the model is:

$$
A_{i}=A_{1}(1-K)^{i-1}(i=1,2,3, \ldots, S) .
$$

Statistical models

The log-normal model, proposed by Preston (1948), considers that the logarithm of the total number of individuals $(\mathrm{N})$ in the community conforms to the normal distribution, and the abundance of the $i$-th species is:

$$
\mathrm{A}_{i}=e^{[\log (\mu)+\log (\sigma) \Phi]}(i=1,2,3, \ldots, S) .
$$

where $\mu$ and $\sigma$ represent the mean and variance of the normal distribution, respectively, and $\Phi$ represents the normal deviation.

The log-series model, proposed by Fisher et al. (1943), is a discrete distribution model. It is suitable for describing positive integers that do not contain 0 , that is, species without individual existence will not be considered. According to this model, the number of species (S) with an abundance of $n$ in the community can be expressed as:

$$
S(n)=\alpha \frac{X^{n}}{n}
$$

where $\alpha$ represents the species diversity of the community, which is similar to the concept of species richness (McGill, 2010). $\mathrm{X}$ is a constant $(0<\mathrm{X} \leq 1)$, related to the size of the community (Zhang et al., 2015).

\section{Neutral theory models}

The metacommunity zero-sum multinomial distribution model assumes that the species abundance distribution at a certain point comes from the random drift of the neutral metacommunity. The model includes two parameters: the number of individuals in the sampling point $(\mathbf{J})$ and the fundamental biodiversity number $(\theta)$. The log-series distribution is a special case of this model (Hubbell, 2001), so the fitting effects of the two models are very similar. According to this model, the number of species (S) with an abundance of $n$ at any sampling point in the metacommunity can be expressed as:

$$
\begin{aligned}
& S(n)=\frac{\theta}{n} \int_{0}^{J} f_{n, 1}(y)\left(1-\frac{y}{J}\right)^{\theta-1} d y \\
& f_{n, \delta}(y)=\frac{1}{\Gamma(n) \delta^{n}} \exp \left(-\frac{y}{\delta}\right) y^{n-1} .
\end{aligned}
$$


The Volkov model adds the parameter migration coefficient $m$ to the previous metacommunity zero-sum multinomial distribution model. It also assumes that the migration coefficient $m$ of the species from the compound community to the local community is fixed. According to this model, the number of species $(\mathrm{S})$ with an abundance of $n$ in a local community can be expressed as:

$$
\begin{aligned}
& S(n)=\theta \frac{J !}{n !(J-n) !} \frac{\Gamma(\gamma)}{\Gamma(J+\gamma)} \int_{0}^{\gamma} \frac{\Gamma(n+y)}{\Gamma(1+y)} \frac{\Gamma(J-n+\gamma-y)}{\Gamma(\gamma-y)} \exp \left(-\frac{y \theta}{\gamma}\right) d y \\
& \Gamma(z)=\int_{0}^{\infty} t^{z-1} e^{-t} d t \\
& \gamma=\frac{m(J-1)}{1-m}
\end{aligned}
$$

where $\gamma$ represents the number of individuals migrating to the local community (Volkov et al, 2003; Zhang Shan et al., 2015).

To test which of the above six models best fit the observed species abundance distributions, we used AIC values and K-S tests. The advantage of AIC is that this approach takes into account both the optimality of model predictions and the simplicity of the model. Models with both a greater similarity between the probability distribution predicted by the model and the real data, and fewer parameters contained in the model, would receive a lower AIC and be determined as the best-fitting model (Burnham \& Anderson, 2002). We also used the K-S test to judge whether there was a significant difference between the two samples by calculating the distance (statistic D) between the empirical distribution function (empirical distribution function) of the two samples.

Spatial autocorrelation detection at different scales

We used Moran's I spatial autocorrelation index to detect whether there was spatial autocorrelation in the species abundance at different spatial scales (Borcard et al., 2014), using the formula

$$
I(d)=\frac{\frac{1}{w} \sum_{h=1}^{n} \sum_{i=1}^{n} w_{h i}\left(y_{h}-\bar{y}\right)\left(y_{i}-\bar{y}\right)}{\frac{1}{n} \sum_{i=1}^{n}\left(y_{i}-\bar{y}\right)^{2}}(h \neq i),
$$

where $y_{h}$ and $y_{i}$ represent the $h$ and $\mathrm{i}$ observations of $\mathrm{y}$, respectively, $\bar{y}$ represents the average value of $y$, and $n$ is the total number of sample squares. When the distance between the sample squares $\mathrm{y}_{\mathrm{h}}$ and $\mathrm{y}_{\mathrm{i}}$ is equal to the step length $\mathrm{d}$, the adjacent weight value $\mathrm{w}_{\mathrm{hi}}=1$, otherwise $\mathrm{w}_{\mathrm{hi}}=0$.

Moran's I index can be used to reflect the similarity of the attribute values of spatially adjacent or spatially adjacent regional units, and its value range is $[-1,1]$. A significant spatial autocorrelation relationship shows that the calculated variables have a non-random spatial distribution pattern. The value tends to 1 , indicating that the overall positive spatial correlation is relatively high, and the distribution of units with similar properties is more concentrated. The value of the index tends to -1 , which indicates that the degree of negative spatial correlation is higher, and the difference between adjacent units is generally larger. The value of Moran's I index close to 0 indicates that the overall spatial autocorrelation degree is low (Qiu et al., 2019).

Distance-based Moran's eigenvector map analysis and variation partitioning

In order to explore the relative importance of niche and neutral processes for community assembly under different sampling scales, we used distance-based Moran's eigenvector map (dbMEM) analysis 
combined with variance decomposition to decompose the relative contributions of environmental factors and spatial structure variables to community composition.

First, we represented the spatial structure variables in this study with distance-based Moran's eigenvector maps (dbMEM), previously called principal coordinate of neighbour matrices (PCNM) (Borcard \& Legendre, 2002). This method can effectively obtain the spatial structure between sample squares at different sampling scales, and is often used to analyze the explanatory variables of spatial change in a community (Borcard et al, 2004; Dray et al, 2006; Smith \& Lundholm, 2010). At each sampling scale, the Euclidean distance matrix is calculated using the center coordinates of each sample square, followed by the spatial structure variables (Legendre et al, 2009; De Cáceres et al, 2012).

In order to reduce the collinearity between the soil and topographical factors, all variables were log-transformed. The degree of collinearity between environmental factors was measured with the Variance Inflation Factor (VIF). A VIF > 10 suggests that there may be a problem of collinearity. In this study, the VIF value of TN exceeded 10 in all three sampling scales and was eliminated.

Before performing variation partitioning, further variable screening of the obtained model is needed to eliminate redundancy. After screening, environmental factors and spatial structure variables that had a significant impact on the response variables can be incorporated into the model for variation partitioning analysis. In this analysis, the response variable was the species abundance distance matrix under each sampling scale, and the explanatory variables were divided into two parts: significant environmental factor variables and significant spatial structure variables. Variation partitioning divides the drivers of variation in species abundance into four parts: (a) simple environmental, (b) spatial structure of the environment, (c) simple spatial, (d) unknown. Of these four, the pure environmental explanation is related to habitat filtering, and the pure spatial explanation is related to dispersal limitation.

All statistical analyses were conducted using R version 4.1.0 (R Core Team, 2021). The species abundance distribution model fitting was completed using the "sads" software package (Paulo et al., 2018). The test of spatial autocorrelation was done using the sp.correlogram function in the "spdep" software package (Bivand et al., 2018). The dbMEM analysis was done using the "adespatial" software package (Stéphane et al., 2021). The variation partitioning analysis is implemented using the varpart function of the "vegan" software package (Jari et al., 2020).

\section{Results}

Patterns of species abundance distributions

A total of 114 species were documented on the entire $200 \times 100 \mathrm{~m}$ plot. The maximum number of species recorded at different sampling scales was 6 species (small $10 \times 10 \mathrm{~m}$ scale), 9 (medium $20 \times 20$ $\mathrm{m}$ ), and 14 (large $50 \times 50 \mathrm{~m}$ ) (Figure 1). At both the small and medium scales, the species abundance distribution of the community presented a monotonically decreasing distribution (Figure 1a and b). At the large sampling scale, a small peak was observed in the interval of 4-8 abundance, reflecting a bimodal distribution (Figure 1c). 
(a) $10 \times 10 \mathrm{~m}$

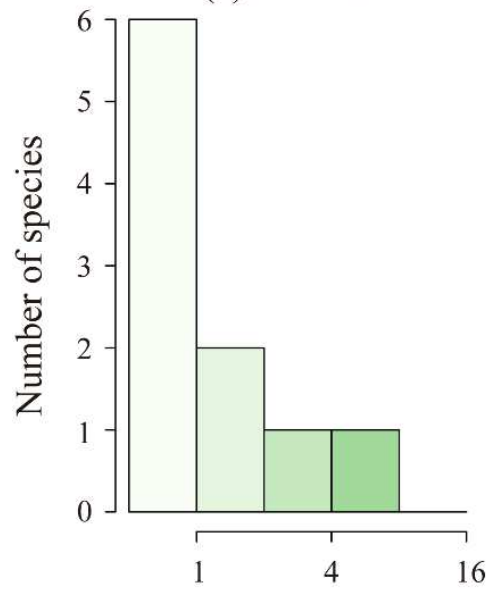

303 model still showed a good fit. (b) $20 \times 20 \mathrm{~m}$

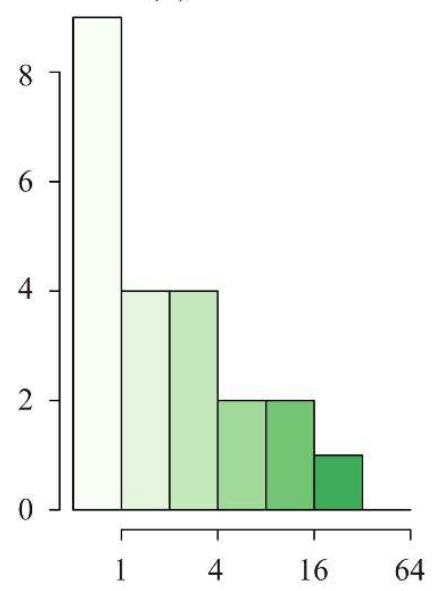

Abundance (c) $50 \times 50 \mathrm{~m}$

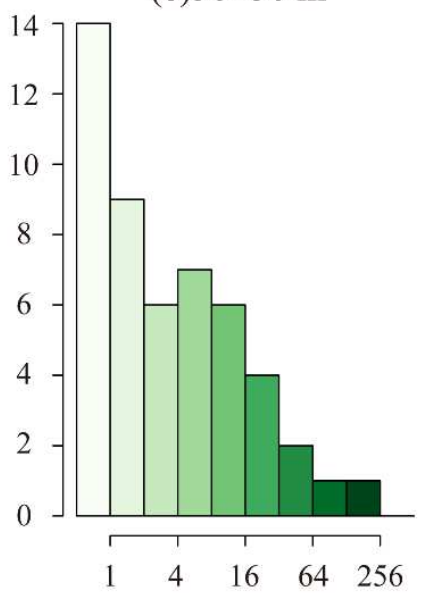

Figure 1. Species-abundance histograms at different scales (a) $10 \times 10 \mathrm{~m}$

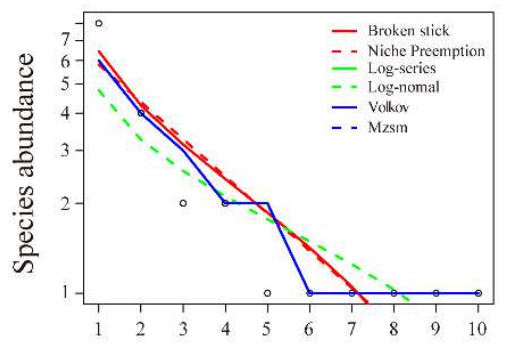

Figure 2. Species-abundance distribution and model fittings at different sampling scales

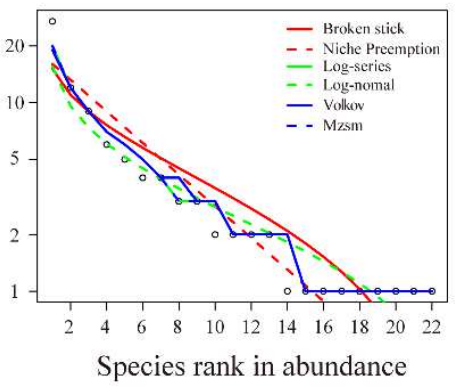

(c) $50 \times 50 \mathrm{~m}$

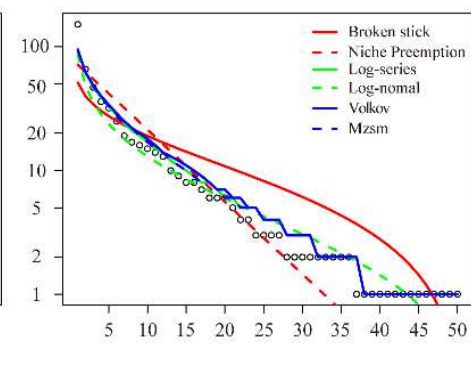

The fitting effect of each model varies with the sampling scale (Figure 2 and Table 1). At the small and medium sampling scales of $10 \mathrm{~m} \times 10 \mathrm{~m}$ and $20 \mathrm{~m} \times 20 \mathrm{~m}$, all models passed the K-S test and were within an acceptable range $(\mathrm{p}>0.05)$. In particular, both the neutral model and the statistical model were more consistent with the data, and the AIC values indicated only a small difference between these two models. At the large sampling scale $(50 \mathrm{~m} \times 50 \mathrm{~m})$, the fit of the two niche models was poor, and the niche preemption model was rejected. However, the neutral model and statistical

Table 1. AIC values and K-S test statistics of 6 models under different sampling scales

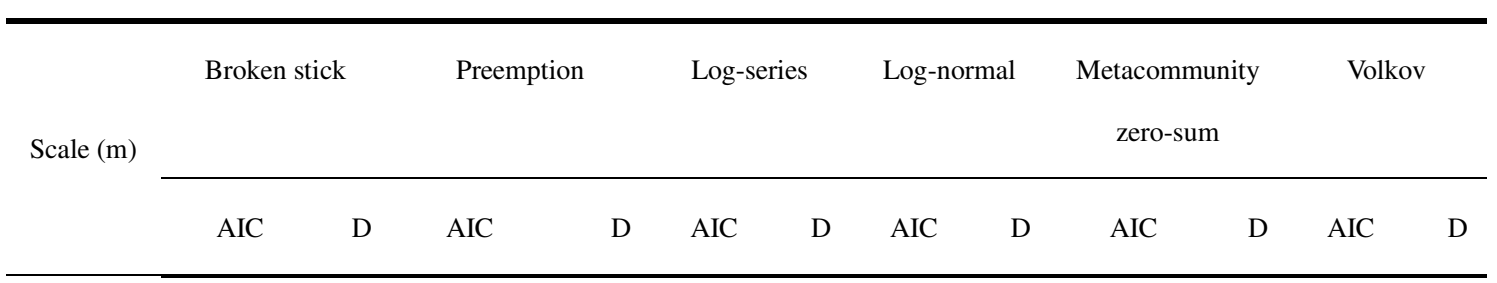




\begin{tabular}{rrrrrrrrrrrrrrr}
\hline $10 \times 10$ & 89.7 & 0.30 & 90.9 & 0.30 & $\mathbf{3 0 . 9}$ & 0.40 & 34.9 & 0.10 & $\mathbf{3 1 . 0}$ & 0.10 & $\mathbf{3 2 . 9}$ & 0.10 \\
$20 \times 20$ & 464.5 & 0.22 & 464.7 & 0.32 & $\mathbf{9 5 . 9}$ & 0.22 & 101.7 & 0.05 & $\mathbf{9 6 . 1}$ & 0.05 & $\mathbf{9 7 . 9}$ & 0.05 \\
& & & & & & & & & & & & & & \\
$50 \times 50$ & 3530.8 & $0.32 *$ & 3414.9 & $0.36^{* * *}$ & $\mathbf{3 0 2 . 8}$ & 0.16 & 314.4 & 0.08 & $\mathbf{3 0 3 . 3}$ & 0.0 & $\mathbf{3 0 4 . 8}$ & 0.08 \\
\hline
\end{tabular}

Note. AIC, Akaike's information criterion; D, K-S test. ${ }^{*} \mathrm{p}<0.05, * * \mathrm{p}<0.01, * * * \mathrm{p}<0.001$.

Spatial autocorrelation detection at different scales

In general, at larger sampling scales, the strength of spatial correlation was weaker (Figure 3). At the sampling scale of $10 \mathrm{~m} \times 10 \mathrm{~m}$, there was significant positive spatial correlation at distances of 1-7, spatially independence at distance levels $8-12$, and significant negative spatial correlation at distance levels 13-20 (Figure 3a). At the sampling scale of $20 \mathrm{~m} \times 20 \mathrm{~m}$, there was positive spatial correlation at distance levels of 1-3, spatial independence at distance levels 4-6 and 10, and negative spatial correlation at distance levels 7-9 (Figure 3b). At a scale of $50 \mathrm{~m} \times 50 \mathrm{~m}$, the spatial pattern showed a weak spatial positive correlation at the distance level of 1 (Figure 3c).
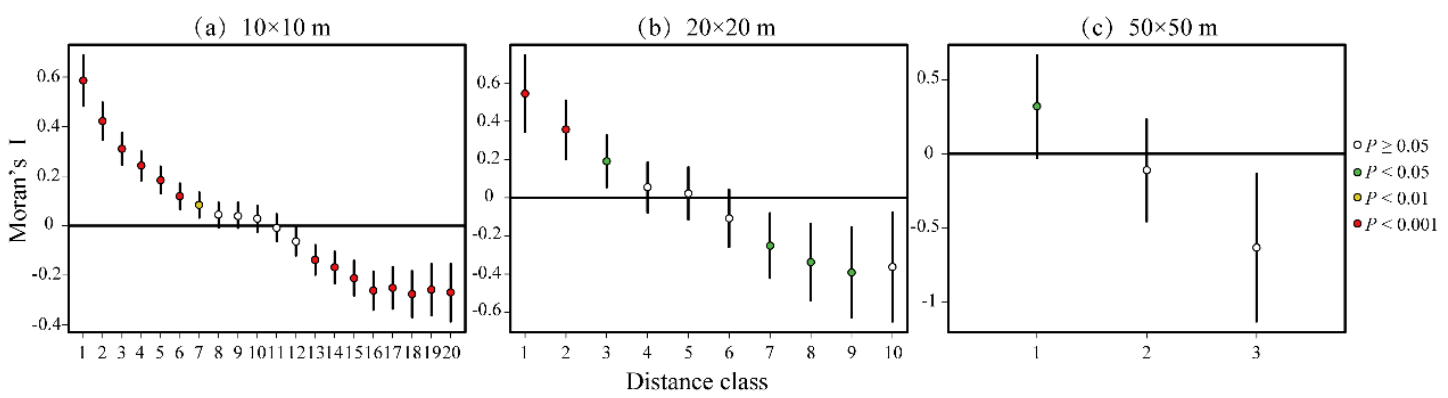

Figure 3. Spatial autocorrelation detection at different scales

Variation partitioning at different scales

The results of the variance partitioning analysis varied with sampling scale (Figure 4). The proportion of variance in the species abundance distribution explained by pure spatial structure was an order of magnitude lower at the largest sampling scale compared to the smallest. Similarly, the proportion of unexplained variation was also lowest at the largest sampling scale. In contrast, the proportion of variance in the species abundance distribution explained by spatial structure of the environment was highest at the largest sampling scale. Curiously, the proportion of variance in the species abundance distribution explained by simple environmental variation was greatest at the medium sampling scale. 
(a) $10 \times 10 \mathrm{~m}$

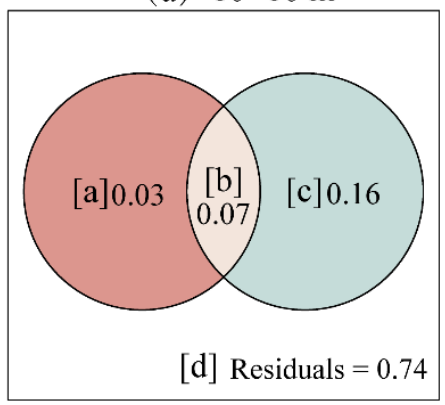

(b) $20 \times 20 \mathrm{~m}$

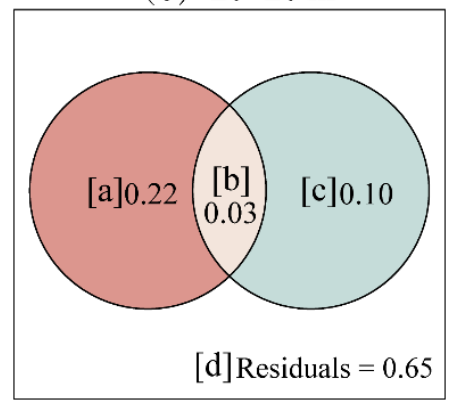

(c) $50 \times 50 \mathrm{~m}$

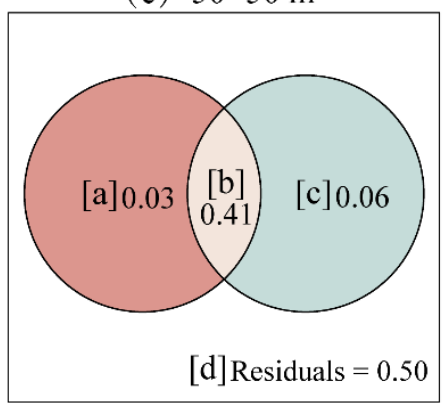

Figure 4. Variation partitioning results of community composition at different scales: [a] simple environmental explanation, [b] spatial structure of the environment, [c] simple spatial explanation, [d] unclear part.

\section{Discussion}

\section{Patterns of species abundance distributions}

At larger sampling scales, the species abundance distribution of the community changed from a monotonous decrease to a bimodal distribution. This result indicates that common species play an important role in forest communities as the sampling scale increases. At the same time, species with an abundance of 1 always occupied the largest proportion of species in each sampling scale, indicating that the status of these rare species in the community cannot be ignored.

The fitting results of the species abundance distribution model of woody plants in the karst evergreen and deciduous broad-leaved mixed forest show that a pure statistical model can describe the species abundance structure and its quantitative relationship at various sampling scales. The species abundance distribution pattern of each community obeys both log-normal distribution and log-series distribution, but it is more inclined to the latter. Many studies have found that these two distribution patterns often appear in different ecological contexts. Immigrant species usually exist in the community in the form of rare species (Zillio and Condit, 2007), so frequent immigration events lead to a complex community composition. There are a large number of rare species, and then show a log-series distribution. Specifically, the metacommunity zero-sum multinomial distribution based on the neutral theory takes into account the immigration rate, and when the immigration rate is 1 , the distribution is consistent with the log-series distribution (Magurran, 2005). The strong dispersal limitation leads to a simple species composition of the community. It is mainly composed of common species, and rare species are scarce, so they exhibit a log-normal distribution. Therefore, the log-series model is also considered a neutral model, and the log-normal model is considered a niche model (Ulrich et al., 2015; Ulrich et al., 2016). This is not completely opposed to the above view, because the reason for the small number of immigrants in the community may be that the selection effect based on niche (such as habitat filtering) reduces the success rate of immigration events. Therefore, from the result that the species abundance curve fits the log-series distribution rather than the lognormal distribution, the neutral process in the karst evergreen and deciduous broad-leaved mixed forest may have a higher explanatory power than the niche process. The fitting effect of the neutral model is better than that of the niche model at all sampling scales, which is also in line with this speculation. In addition, at small 
and medium scales $(10 \mathrm{~m} \times 10 \mathrm{~m}$ and $20 \mathrm{~m} \times 20 \mathrm{~m})$, both the niche model and the neutral model have a good fit, indicating that the niche process and the neutral process work together in community assembly at the small and medium scales. However, at a large scale $(50 \mathrm{~m} \times 50 \mathrm{~m})$, the fitting effect of the niche model deteriorated significantly and was finally rejected. In contrast, the neutral theoretical model can better fit the abundance distribution of woody plants in karst evergreen and deciduous broad-leaved mixed forest at all scales. Based on the above results, it is indicated that as the sampling scale increases, the neutral process may be more important than the niche process in maintaining community construction.

The relative importance of niche process and neutral process

For a long time, the species abundance distribution pattern has been considered to reflect the species diversity maintenance mechanism of the community to a certain extent (Tokeshi, 1993; Hubbell, 2001; Volkov et al., 2003). However, it is worth noting that a specific species abundance distribution can be caused by different processes or mechanisms (Chisholm and Pacala, 2010; Matthews et al., 2017). Therefore, when interpreting the distribution of species abundance, it is necessary to combine the actual status of the community, but also to distinguish the theoretical background corresponding to the specific pattern. This study uses spatial autocorrelation and dbMEM combined with variation partitioning methods to further measure the relative effects of niche processes and neutral processes at different spatial scales, and more comprehensively reveal the underlying mechanism of the formation of species composition differences among communities.

The spatial autocorrelation results show that the species abundance of the evergreen and deciduous broad-leaved mixed forest community has a significant spatial autocorrelation at a small sampling scale of $10 \mathrm{~m} \times 10 \mathrm{~m}$. At the medium sampling scale of $20 \mathrm{~m} \times 20 \mathrm{~m}$, the spatial autocorrelation relationship is weakened. At a large sampling scale of $50 \mathrm{~m} \times 50 \mathrm{~m}$, the spatial autocorrelation relationship is already very weak. As the sampling scale increases, the tendency of the spatial correlation strength to gradually weaken indicates that the interaction between species is gradually weakening as the sampling scale increases. This is consistent with the conclusions of most previous studies. The contribution of interactions between species to community construction is more concentrated in small and medium scales. In addition, as the distance level gradually increases, the spatial structure exhibits a significant spatial positive correlation to a spatially irrelevant relationship, then a significant spatial negative correlation relationship, and even a greater distance may show a spatial positive correlation relationship. This may be due to the strong aggregation and distribution of dominant species in karst forests and the patchy distribution of soil within a small distance, resulting in a patchy spatial structure in the distribution of species abundance. As the distance increases beyond the patch area, the spatial correlation strength gradually weakens or even becomes irrelevant. Due to the large differences in vegetation composition in different areas of the karst forest (such as different slope positions), when the spacing exceeds a certain distance, there are generally large differences in abundance between different squares, which is a negative spatial correlation. When the distance between different squares is larger but the habitat conditions are more similar, the community abundance may have a positive spatial correlation (Wang et al., 2014).

The results of variation partitioning show that as the sampling scale becomes larger, the interpretation rate of the spatial structure gradually decreases. This is also consistent with the result that the fitting effect of the neutral theoretical model becomes worse as the sampling scale becomes larger. 
However, the interpretation rate of the simple spatial structure at each sampling scale occupies an important proportion, which indicates that the neutral process is a driving force that cannot be ignored in the process of community construction at each sampling scale. The pure environmental explanation was only $3 \%$ at the small sampling scale, but increased to $22 \%$ at the medium sampling scale. This increase may be related to the low environmental heterogeneity among plots at small scales. As the sampling scale increases, environmental heterogeneity also gradually increases. However, under niche theory, habitat filtering and interspecies competition are two driving forces that act in opposite directions in the process of community assembly (Yan et al., 2010; Chai \& Yue, 2016). The coexistence of these two forces will weaken the contribution of the niche process to the species abundance pattern of the community. Therefore, at small scales, it is subject to strong inter-species competition and weak habitat filtering, and the niche process also contributes higher to the species abundance pattern of the community. At the medium scale, these two forces are both strong, and when the two offset each other, the contribution of the niche process to the species abundance pattern of the community is lower. Both of these two forces are weak when detected at a large scale, and the niche model is not as effective as the neutral model in fitting the community species abundance pattern (Jones et al, 2008; Legendre et al, 2009; Yao et al., 2020). Therefore, in general, the effect of habitat filtering becomes stronger as the sampling scale increases. However, on a large scale, the spatial structure of environmental factors may lead to a pattern of species abundance, which is more in line with the neutral theoretical model. In addition, at each sampling scale, a considerable part of the variation is not explained by known factors. This part may contain some biological and non-spatial structural attributes that are not observed in this study. At the same time, some ecological processes such as the interaction between species have not been considered in the variance decomposition. The spatial autocorrelation test shows that the interaction between species has a strong force at the small and medium sampling scales, which may be the reason for the higher unknown components at the small and medium sampling scales. In this study, limited by the steep terrain of the karst forest community in southwestern China, it is difficult to carry out a large-scale community survey. Therefore, the community construction mechanism at a large scale needs to be verified by more abundant community data in the future.

\section{Conclusions}

In summary, the ecological functions and processes of the karst evergreen and deciduous broad-leaved mixed forest community are a very complex comprehensive effect. Although the above-mentioned research cannot fully reveal its potential information, the forces of the niche process and the neutral process at each sampling scale have been strongly verified. The two interact and jointly dominate the construction process of the forest community in this area. Therefore, in the restoration and reconstruction of karst evergreen and deciduous broad-leaved mixed forest communities in southwestern China, environmental heterogeneity, inter-species relationships and geographic spatial

\section{Acknowledgements}

This research was jointly supported by the National Natural Science Foundation of China (31860124) 
and by the Guangxi Normal University Scientific Research and Education Special Project (Natural Science) (RZ2100000150).

\section{Author contributions}

Y.J. and S.L. designed and oversaw the study; H.L., Y.P., Y.H., Y.F., W.Z. and Y.X. collected the field and laboratory data; Y.H. and Y.J. conducted the statistical analyses and wrote the first draft of the manuscript; all authors contributed critically to the drafts and approved the final manuscript.

\section{Declarations}

Declaration of competing interest The authors declare that they have no known competing financial interests or personal relationships that could have appeared to influence the work reported in this paper.

Informed consent All authors gave consent for conducting and publishing the research.

Research involving human participants and/or animals No human participants or animals were involved in the present research.

\section{References}

Agricultural Chemistry Professional Committee of Chinese Soil Society. Routine Analysis Methods of Soil Agricultural Chemistry. Beijing: Science Press, 1983.

Bivand, Roger S. and Wong, David W. S. (2018) Comparing implementations of global and local indicators of spatial association TEST, 27(3), 716-748. https://doi.org/10.1007/s11749-018-0599-x

Borcard D, Legendre P. (2002). All-scale spatial analysis of ecological data by means of principal coordinates of neighbour matrices. Ecological Modelling, 153, 51-68. https://doi.org/10. 1016/S0304-3800(01)00501-4

Borcard D, Legendre P, Avois-Jacquet C, Tuomisto H. (2004). Dissecting the spatial structure of ecological data at multiples scales. Ecology, 85, 1826-1832.

Borcard D, Gillet F, Legendre P, Authors. Lai Jiangshan, Trans. (2014). Quantitative Ecology Application of R Language. Beijing: Higher Education Press, 206-208

Burnham KP, Anderson DR. (2002). Model Selection and Multi-Model Inference. A Practical Information-Theoretic Approach, 2nd edn. Springer-Verlag, New York.

Chai Y F, Yue M.(2016). Research advances in plant community assembly mechanisms. Acta Ecologica 
Cheng Jiajia, Mi Xiangcheng, Ma k-Ping \& Zhang Jintun. (2011). Responses of species-abundance distribution to varying sampling scales in a subtropical broad-leaved forest. Biodiversity Science, 19(02), 168-177. https://doi.org/10.3724/SP.J.1003.2011.10107

Chisholm R A, Pacala S W. (2010). Niche and neutral models predict asymptotically equivalent species abundance distributions in high-diversity ecological communities. Proceedings of the National Academy of Sciences of the United States of America, 107(36): 15821-15825. https://doi.org/10.1073/pnas.1009387107

De Cáceres M, Legendre P, Valencia R, Cao M, Chang LW, Chuyong G, Condit R, Hao ZQ,Hsieh CF, Hubbell S, Kenfack D, Ma KP, Mi XC, Noor MNS, Kassim AR, Ren HB, Su SH, Sun IF, Thomas D, Ye WH, He FL. (2012). The variation of tree beta diversity across a global network of forest plots. Global Ecology and Biogeography, 21, 1191-1202. https://doi.org/10.1111/j.14668238.2012.00770.x

Dray S, Legendre P, Peres-Neto PR. (2006). Spatial modelling: A comprehensive framework for principal coordinate analysis of neighbour matrices (PCNM). Ecological Modelling, 196, 483-493. https://doi.org/10.1016/j.ecolmodel.2006.02.015

Fisher RA, Corbet AS, Williams CB. (1943). The relation between the number of species and the number of individuals in a random sample of an animal population. Journal of Animal Ecology, 12, $42-58$.

Furniss T, Larson A, Lutz J. (2017). Reconciling niches and neutrality in a subalpine temperate forest. Ecosphere. 8: e01847. https://doi.org/10.1002/ecs2.1847

Gravel D, Canham C D, Beaudet M, Messier C. (2006). Reconciling niche and neutrality: the continuum hypothesis. Ecology Letters. 9(4): 399-409. https://doi.org/10.1111/j.14610248.2006.00884.x

Green JL. (2007). A statistical theory for sampling species abundances. Ecology Letters, 10, 10371045. https://doi.org/10.1111/j.1461-0248.2007.01101.x

Harms KE, Condit R, Hubbell SP, Foster RB. (2001). Habitat associations of trees and shrubs in a 50ha neotropical forest plot. Journal of Ecology, 89, 947-959.

Hubbell SP. (2001). The unified neutral theory of biodiversity and biogeography. Princeton University Press, Princeton, New Jersey, USA. 
https://doi.org/10.1111/j.1365-2745.2001.00615.x

Hubbell SP, Borda-De-Água L. (2004). The unified neutral theory of biodiversity and biogeography: reply. Ecology, 85, 3175-3178. https://doi.org/10.1890/04-0808

Izsák J, Pavoine S. (2012). Links between the species abundance distribution and the shape of the corresponding rank abundance curve. Ecological indicators. 14(1): 1-6. https://doi.org/10.1016/j.ecolind.2011.06.030

Jari Oksanen, F. Guillaume Blanchet, Michael Friendly, Roeland Kindt, Pierre Legendre, Dan McGlinn, Peter R. Minchin, R. B. O'Hara, Gavin L. Simpson, Peter Solymos, M. Henry H. Stevens, Eduard Szoecs and Helene Wagner (2020). vegan: Community Ecology Package. R package version 2.5-7. https://CRAN.R-project.org/package=vegan

Jones MM, Tuomisto H, Borcard D, Legendre P, Clark DB, Olivas PC. (2009). Explaining variation in tropical plant community composition: Influence of environmental and spatial data quality. Oecologia, 155, 593-604. https://doi.org/10.1007/s00442-007-0923-8

Kong Lei, Yang Hua, Kang Xingang, Gao Yan \& Feng Qixiang. (2011). Review on the methods of spatial distribution pattern in forest. Journal of Northwest Sci-Tech University of Agriculture and Forestry (Natural Science) (05), 119-125. https://doi.org/10.13207/j.cnki.jnwafu.2011.05.032

Legendre P, Mi XC, Ren HB, Ma KP, Yu MJ, Sun IF, He FL. (2009). Partitioning beta diversity in a subtropical broad-leaved forest of China. Ecology, 90, 663-674. https://doi.org/10.1890/07-1880.1

Lei Liqun. (2014). Relationship between community structure and environmental factors in different successional stages of the karst vegetation in Mashan in Guangxi. (Master's thesis, Guangxi University).

Li Xiankun, Jiang Zhongcheng, Lu Shihong, Ou Zulan, Xiang Wusheng, Lu Shuhua \& Su Zongming. (2004). Karst vegetation and its diversity in Guangxi. (eds.) Proceedings of the Third Guangxi Youth Academic Conference (Natural Science) Article) (pp.771-774). Guangxi People's Publishing House.

Li Qiao, Tu Jing, Xiong Zhongping, Lu Zhixing, Liu Chunju. (2011). Survey of research on species abundance pattern. Journal of Yunnan Agricultural University (Natural Science) (01), 117-123. https://doi.org/10. 3969/j.issn.1004-390X(n).2011.01.021 
Liu Runhong, Bai Jinlian, Bao Han, Nong Juanli, Zhao Jiajia, Jiang Yong, Liang Shichu, Li Yuejuan. (2020). Variation and correlation in functional traits of main woody plants in the Cyclobalanopsis glauca community in the karst hills of Guilin, southwest China. Chinese Journal of Plant Ecology, 44(08):828 -841. https://doi.org/10.17521/cjpe.2019.0146

Magurran A E. (2004). Measuring Biological Diversity. Oxford: Blackwell Science.

Magurran A E. (2005). Species abundance distributions: pattern or process? Functional Ecology, 19(1): 177-181. https://doi.org/10.1111/j.0269-8463.2005.00930.x

Ma Ke-Ming. (2003). Advance of the study on species abundance pattern. Chinese Journal of Plant Ecology (03), 412-426.

Mac Arthur R H. (1957). On the relative abundance of bird species. Proceedings of the National Academy of Sciences of the United States of America. 43(3): 293-295. https://doi.org/10.1073/pnas.43.3.293

Matthews T J, Whittaker R J. (2015). On the species abundance distribution in applied ecology and biodiversity management. Journal of Applied Ecology. 52(2): 443-454. https://doi.org/10.1111/1365-2664.12380

Matthews T J, Borges P A V, de Azevedo E B, Whittaker R J. (2017). A biogeographical perspective on species abundance distributions: recent advances and opportunities for future research. Journal of Biogeography, 44(8): 1705-1710. https://doi.org/10.1111/jbi.13008

McGill BJ. (2003). Does Mother Nature really prefer rare species or are log-left-skewed SADs a sampling artifact? Ecology Letters, 6, 766-773. https://doi.org/10.1046/j.1461-0248.2003.00491.x

McGill BJ, Etienne RS, Gray JS, Alonso D, Anderson MJ, Benecha HK, Dornelas M, Enquist BJ, Green JL, He FL, Hurlbert AH, Magurran AE, Marquet PA, Maurer BA, Ostling A, Soykan CU, Ugland KI, White EP. (2007). Species abundance distributions: moving beyond single prediction theories to integration within an ecological framework. Ecology Letters, 10, 995-1015. https://doi.org/10.1111/j.1461-0248.2007.01094.x

McGill BJ. (2010). Species abundance distributions. In: Bio-logical Diversity: Frontiers in Measurement and Assessment (eds Magurran AE, McGill BJ). Oxford University Press, New York. Motonura I. (1932). On the statistical treatment of communities. Zoological Magazine (Tokyo), 44, 379-383. 
Niu Kechang, Liu Yining, Shen Zehao, He Fangliang, Fang Jingyun. (2009). Community assembly: the relative importance of neutral theory and niche theory. Biodiversity, 17(06): 579-593. https://doi.org/doi:10.3724/SP.J.1003.2009.09142

Pan Yuanfang, Liang Zhihui, Li Jiabao, Liang Shichu, Jiang Yong, Wu Huaping, Wang Jingjing, Fu Ruijing, Zhou Jianmei. (2021). Community structure and species diversity of evergreen and deciduous broad-leaved mixed forests in Karst hills of Guilin. Acta Ecologica Sinica, 41(06):2451-2459. http://dx.doi.org/10.5846/stxb201906031169

Paulo I. Prado, Murilo Dantas Miranda and Andre Chalom (2018). sads: Maximum Likelihood Models for Species Abundance Distributions. $\mathrm{R}$ package version 0.4.2. https://CRAN.Rproject.org/package $=$ sads

Preston FW. (1948). The commonness, and rarity, of species. Ecology, 29, 254-283. https://doi.org/10.2307/1930989

Qiu Y N, Ren S Y, Wang X, Yang P H, Li Y Y, Li S H, Wu X L, Wu S H, Xu Z W, Li G Q, Huang C, Xu C. (2019). The spatial dynamics of vegetation revealed by unmanned aerial vehicles images in a straw-checkerboards-based ecological restoration area. Acta Ecologica Sinica, 39(24):9058-9067. http://dx.doi.org/10.5846/stxb201810172245

R Core Team (2021). R: A language and environment for statistical computing. R Foundation for Statistical Computing, Vienna, Austria. https://www.R-project.org/.

Shipley B, Paine C E T, Baraloto C. (2012). Quantifying the importance of local niche-based and stochastic processes to tropical tree community assembly. Ecology. 93(4): 760-769. https://doi.org/10.1890/11-0944.1

Smith TW, Lundholm JT. (2010). Variation partitioning as a tool to distinguish between niche and neutral processes. Ecography, 33, 648-655. https://doi.org/10.1111/j.1600-0587.2009.06105.x

Stéphane Dray, David Bauman, Guillaume Blanchet, Daniel Borcard, Sylvie Clappe, Guillaume Guenard, Thibaut Jombart, Guillaume Larocque, Pierre Legendre, Naima Madi and Helene H Wagner (2021). adespatial: Multivariate Multiscale Spatial Analysis. R package version 0.3-14. https://CRAN.R-project.org/package=adespatial

Tokeshi M. (1993). Species Abundance Patterns and Community Structure. In: Begon M, Fitter A H (eds.), Advances in Ecological Research. volume 111-186. Academic Press. https://doi.org/10.1016/S0065-2504(08)60042-2 
Tokeshi M. (1996). Power fraction: a new explanation of relative abundance patterns in species-rich assemblages. Oikos. 75: 543-550. https://doi.org/10.2307/3545898

Ulrich W, Kusumoto B, Shiono T, Kubota Y. (2015). Climatic and geographic correlates of global forest tree species-abundance distributions and community evenness. Journal of Vegetation Science. 27(2): 295-305. https://doi.org/10.1111/jvs.12346

Ulrich W, Soliveres S, Thomas A D, Dougill A J, Maestre F T. (2016). Environmental correlates of species rank-abundance distributions in global drylands. Perspectives in plant ecology, evolution and systematics, 20: 56-64. https://doi.org/10.1016/j.ppees.2016.04.004

Valencia R, Foster RB, Villa G, Condit R, Svenning JC, Hernández C, Romoleroux K, Losos E, Magård E, Balslev H. (2004). Tree species distributions and local habitat variation in the Amazon: Large forest plot in eastern Ecuador. Journal of Ecology, 92, 214-229. https://doi.org/10.1111/j.0022-0477.2004.00876.x

Vellend M, Srivastava D S, Anderson K M, Brown C D, Jankowski J E, Kleynhans E J, Kraft N J B, Letaw A D, Macdonald A A M, Maclean J E, et al. (2014). Assessing the relative importance of neutral stochasticity in ecological communities. Oikos. 123(12): 1420-1430. https://doi.org/10.1111/oik.01493

Volkov I, Banavar JR, Hubbell SP, Maritan A. (2003). Neutral theory and relative species abundance in ecology. Nature, 424, 1035-1037. https://doi.org/10.1038/nature01883

Wang Bin, Xiang Wusheng, Ding Tao, Huang Fuzhao, Wen Shujun, Li Dongxing, Guo Yili, Li Xiankun. (2014). Spatial distribution of standing dead trees abundance and its impact factors in the karst seasonal rain forest, Nonggang, southern China (in Chinese). Chin Sci Bull (Chin Ver), 59: 3479_3490.

Wang Shixiong, Guo Hua, Wang Xiaoan \& Fan Weiyi. (2013). Dispersal limitation versus environment filtering in the assembly of plant communities in the Ziwu Mountains. Scientia Agricultura Sinica, (22), 4733-4744. https://doi.org/10.3864/j.issn.0578-1752.2013.22.011

Wiegand T, Uriarte M, Kraft N, Shen G, Wang X, He F. (2017). Spatially Explicit Metrics of Species Diversity, Functional Diversity, and Phylogenetic Diversity: Insights into Plant Community Assembly Processes. Annual review of ecology and systematics. 48: 329-351. https://doi.org/10.1146/annurev-ecolsys-110316-022936

Wu A, Deng X, he H, Ren X, Yiran, Xiang W, Shuai O, Yan W, Fang X. (2019). Responses of species 
abundance distribution patterns to spatial scaling in subtropical secondary forests. Ecology and Evolution. 9(9), 5338-5347. https://doi.org/10.1002/ece3.5122

Wu Manling, Zhu Jiang, Zhu Qiang, Huang Xiao, Wang Jin \& Liu Yi. (2019). Analysis of Leaf Functional Traits and Functional Diversity of Woody Plants in Evergreen and Deciduous Broadleaved Mixed Forest of Xingdoushan. Acta Botanica Boreali-Occidentalia Sinica, 39(09), 16781691. https://doi.org/10.7606/j.issn.1000-4025.2019.09.1678

Yan Bangguo. (2010). Research on plant community assembly rules across alpine meadow-forest gradients in Western Sichuan. (Master's thesis, Sichuan Agricultural University).

Yao Zhiliang, Wen Handong, Deng Yun, Cao Min \& Lin Luxiang. (2020). Driving forces underlying the beta diversity of tree species in subtropical mid-mountain moist evergreen broad-leaved forests in Ailao Mountains. Biodiversity $\quad$ Science, 28(04), 445-454. https://doi.org/10.17520/biods.2019356

Zhang S, Lin F, Yuan ZQ, Kuang X, Jia SH, Wang YY, Suo YY, Fang S, Wang XG, Ye J, Hao ZQ. (2015). Herb layer species abundance distribution patterns in different seasons in an old-growth temperate forest in Changbai Mountain, China. Biodiversity Science, 23, 641-648. https://doi.org/10.17520/biods.2015089

Zhang S, Zang R, Huang Y, Ding Y, Huang J, Lu X, Liu W, Long W, Zhang J, Jiang Y. (2016). Diversity maintenance mechanism changes with vegetation type and the community size in a tropical nature reserve. Ecosphere. 7(10): e01526. https://doi.org/10.1002/ecs2.1526

Zillio T, Condit R. (2007). The impact of neutrality, niche differentiation and species input on diversity and abundance distributions. Oikos. 116(6): 931-940. https://doi.org/10.1111/j.00301299.2007.15662.x 